\title{
POPULARIZATION OF ASTRONOMY IN SOUTH INDIA
}

\section{R. Ramakarthikeyan}

Department of Mathematics, RKM Vivekananda College, Madras, S. India, 600004

I am presenting this paper only with reference to my city of Madras, the capital of Tamil Nadu, where more than 60 per cent of the population are illiterate.

$\mathrm{I}$ am an active member of the Madras Astronomical Association and $\mathrm{I}$ am the Vice-President of the Vivekananda Astronomy Club, both of which are popularizing astronomy among the masses.

Though we have been pleading for the necessity of a planetarium over the last two decades, we got one only in May 1988. But for the last seven years, we designed and set up a mini-planetarium at the Education Pavilion of the Trade Fair Exhibition conducted by the local government every year.

In this pavilion we showed the various kinds of eclipses and certain zodiacal constellations using an overhead projector. We did this to eradicate superstitions and to teach the public some basic concepts on astronomy. The response to these programs has been very encouraging. I have also given popular talks on astronomy in several schools in this city and helped them in starting astronomy clubs. We have given a number of television programs on the popularization of astronomy, broadcast statewide. I must mention here that astronomy is not in the curriculum for the state-government-aided schools.

For students and for the public we conduct regular observations at my college astronomy club. In addition to the regular observations, we have also shown the public and students the following celestial phenomena.

1. Comet Kohoutek in Winter 1975 ;

2. the grand spectacle of five planets arrayed within a longitude of $90^{\circ}$ in March 1983 ;

3. several eclipses during the decade;

4. the transit of Mercury on November 13, 1986;

5. Comet Halley in March 1986; and

6. meteor showers.

We have also conducted seminars and exhibitions at regular intervals.

On February 16, 1980, hundreds of students and others attached to the astronomy clubs of various colleges and the members of the Madras Astronomical Association observed the total solar eclipse at Hubli, 500 miles from Madras. In this grand spectacle, we observed at 2:45 p.m.: 1. Baily's beads; 2 . diamond rings; 3. the planets Mercury and Venus; 4. the star Sirius; and 5. shadow bands, and so on. 
We now have plans to tap the talented school children who are interested in astronomy and give them training on various aspects of this subject. The Birla Planetarium attracts thousands of citizens to its shows on astronomy, and we hope that the day is not far off when every citizen of this part of India is aware of the basic concepts of astronomy.

\title{
A SERIES OF ASTRONOMY PROGRAMS FOR TELEVISION IN INDIA
}

\author{
Jayant V. Narlikar \\ Tata Institute of Fundamental Research, Bombay 400005, India \\ and \\ Inter-University Centre for Astronomy and Astrophysics, Pune 411007, India
}

\section{Introduction}

Astronomy, unlike most other sciences, arouses great curiosity amongst laypeople. It is a subject that can be described relatively easily in public lectures. Distinguished astronomers like James Jeans and Arthur Eddington in the past and many more in recent times have "stooped down" to the public level to share the excitement of astronomical discoveries. Today, the popularization program normally proceeds in four different ways - through popular articles, public lectures, planetarium shows, and radio - TV programs. However, this overwhelming public interest in astronomy brings its own difficulties. Not all of it is motivated by a scientific interest! Many persons read mystic significance into astronomical findings. Many more are guided by astrological interest. Many fail to perceive the scientific basis for astronomy, a subject whose laboratory is the whole cosmos with objects too remote to be subject to scientific experimentation.

Thus "stooping down" to the public level is not so easy! In fact, I consider it more difficult to prepare a public lecture on an astronomical topic than to give a technical talk on a research problem. After all, in the former case one has to surmount the above-mentioned difficulties that don't exist in the latter case. It is not surprising, therefore, that even today the number of really successful popularizers of the subject is small.

Yet, wherever successful, the rewards of such efforts have been great. The books and lectures of Jeans and Eddington thrilled an entire generation of educated public before the Second World War. I know several of my own generation who drew inspiration from Fred Hoyle's lectures on BBC radio that were subsequently published as the book The Nature of the Universe. 